\title{
Measurement of cross sections and couplings of the SM Higgs boson in the WW decay channel using the ATLAS detector
}

\author{
Claudia Bertella ${ }^{* \dagger}$ \\ Johannes-Gutenberg-Universitaet Mainz (DE) \\ E-mail: claudia.bertella@cern.ch
}

\begin{abstract}
The measurement of the coupling strength of the Higgs boson to the two vector bosons using the $H \rightarrow W W \rightarrow l v l v$ channel is presented using the $25 \mathrm{fb}^{-1}$ of data collected at 7 and $8 \mathrm{TeV}$ center-of-mass energy by the ATLAS experiment. The extractions of the signal yields in the three major production modes, gluon fusion (ggF), vector boson fusion (VBF) and Higgs-strahlung, are presented as well as the final combination. Moreover, the measurement of fiducial and differential cross sections of gluon-fusion Higgs boson production is presented and discussed upon.
\end{abstract}

38th International Conference on High Energy Physics

3-10 August 2016

Chicago, USA

* Speaker.

${ }^{\dagger}$ on behalf of the ATLAS Collaboration 


\section{Introduction}

The LHC collider delivered proton-proton collisions at a center-of-mass energy of $7 \mathrm{TeV}$ and 8 $\mathrm{TeV}$ allowing the ATLAS experiment [1] to collected about $25 \mathrm{fb}^{-1}$ during 2011 and 2012. One of the main focus of the Collaboration was to search for the Higgs boson decaying in vector bosons, since the non-vanishing coupling to vector boson is a consequence of the electro-weak symmetry breaking. The $H \rightarrow W W^{*} \rightarrow \ell v \ell v$ decay is characterized by a large branching ratio and a clear signature identified by selecting two isolated leptons with opposite charge $(e, \mu)$ in any flavour combination and having transverse momenta satisfying the following selection: $p_{\mathrm{T}}^{\text {lead }}>25 \mathrm{GeV}$ and $p_{\mathrm{T}}^{\text {sub-lead }}>15 \mathrm{GeV}$. The $H \rightarrow W W^{*} \rightarrow \ell v \ell v$ has been investigated via gluon fusion, vectorboson-fusion and vector boson associate $(\mathrm{VH})$ production.

\section{Gluon fusion and vector boson fusion production modes}

The ggF and VBF production modes are maily distinguished by the associated jet multiplicity [2]. Also, the dominant backgrounds depend on the jet multiplicity. Therefore jet multiplicity categories have been defined to enhance the significance. For $N_{j e t} \leq 1$, the $W W$ and the top-quark background are dominant while the top-quark background is the most dominant for $N_{\text {jet }} \geq 2$. The number of jets identified by a b-tagging algorithm $\left(N_{b-j e t}\right)$ are used to veto events with jets coming from a b-quark for $N_{j e t} \geq 1$. A set of topological selections have been defined in order to take advantage of the $V-A$ structure of the $H \rightarrow W W^{*}$ decay, which leads to a spin correlation between the two leptons. The invariant mass of the leptons, $m_{l l}$, and the azimuthal angle $\Delta \phi_{l l}$ are expected to be smaller for the signal processes with respect to the SM background. In the $N_{\text {jet }}=0$ analysis the transverse momentum of the dilepton system is required to be larger than $30 \mathrm{GeV}$ and the azimuthal gap between $p_{\mathrm{T}}^{l l}$ and the missing transverse momentum $E_{T}^{\text {miss }}$ smaller than $\pi / 2$. In the $N_{\text {jet }} \geq 1$ category, the top-quark background is suppressed by vetoing the events with at least 1 jet identified by the b-tagging algorithm. In the $N_{\text {jet }} \geq 2 \mathrm{VBF}$ enriched region, the kinematics of the two highest jets (tagged jets) in the events and of the Higgs boson system are used in order to define a specific event selection. The analysis is based on a multivariate technique using eight variables, as the invariant mass and the rapidity gap of the tagged jets, in the training in order to separate the signal from the background.

\subsection{Background estimation}

The $W W$ process is an irreducible background for all jet-categories, particularly it is the dominant background for $N_{j e t}=0$. The predicted distributions of the $W W$ background in $N_{j e t}=0$ and 1 are normalized using an enriched orthogonal control region (CR) while it is full estimated by the $\mathrm{MC}$ for $N_{\text {jet }} \geq 2$. Fig. 1a shows the transverse mass in the $W W \mathrm{CR}$. The top-quark background for $N_{\text {jet }}=0$ is estimated using an inclusive-jet region; the extrapolation parameter is defined by the fraction of events with zero reconstructed jets and is derived from the MC simulation. For $N_{\text {jet }} \geq 1$, the top-quark background is normalized in the $\mathrm{CR}$ requiring one $b$-tagged jet. Fig. $1 \mathrm{~b}$ shows the $m_{j j}$ distribution in the top-quark CR used for the VBF category. The $W+$ jets background shape and normalization are estimated from data. The $W+$ jets background in SR is obtained by scaling the 


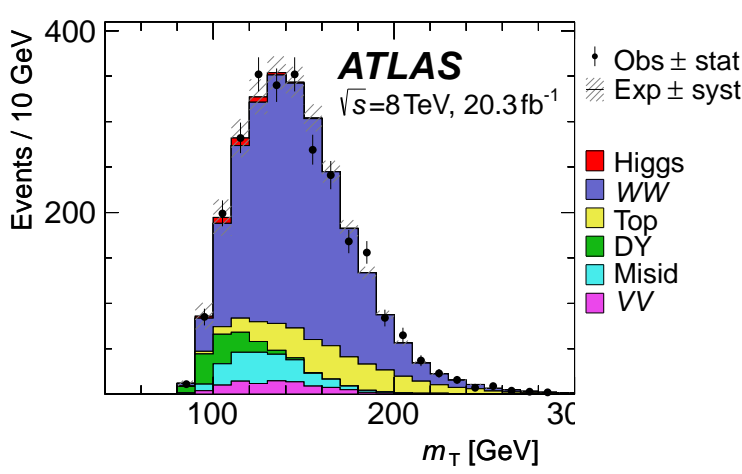

(a) $W W \mathrm{CR}$

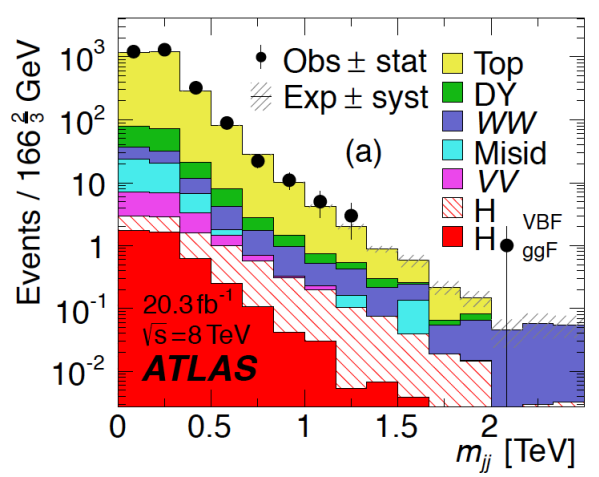

(b) top-quark CR

Figure 1: 1a Transverse mass distribution in the $W W \mathrm{CR}$ distributions. $1 \mathrm{~b}$ Invariant mass of the tagged jets, $m_{j j}$, in the top-quark CR distribution for VBF-enriched $N_{j e t} \geq 2$ category. [2]

number of events in the data $\mathrm{CR}$ by the fake factor $(\mathrm{FF})$ that is defined as the ratio of the number of lepton candidates passing all lepton identification selections to the ones failing the selections.

\subsection{Results}

The combination of the 2011 and 2012 data in all categories shows a clear excess of signal over the background, see Fig. 2a. For the VBF-enriched category, a selection-based analysis, which uses the $m_{\mathrm{T}}$ distribution as the discriminant, is used as a cross-check of the BDT result. Fig. 2b shows the $m_{\mathrm{T}}$ distribution in the upper part and the scatter plot of $m_{j j}$ versus $m_{\mathrm{T}}$. The areas with the highest signal-to-background ratio are characterized by low $m_{\mathrm{T}}$ and high $m_{j j}$. A profile likelihood is used to search for a signal and characterize the production rate in the $\mathrm{ggF}$ and VBF modes. Observation of the inclusive Higgs boson signal with 5.8 standard deviations and evidence for the VBF production mode with 3.2 standard deviations have been established. The combined Higgs boson signal strength, including all the signal region categories, is $\mu=1.09_{-0.21}^{+0.23}$

The PDF variation from the ggF process represents one of the main systematic uncertainties, followed by the QCD re-normalisation and the $W W$ generator modelling. The VBF analysis is, instead, highly affected by the QCD scale uncertainties on the inclusive $N_{j e t} \geq 2$ and $N_{\text {jet }} \geq 3$ cross sections

\section{Higgs-strahlung analysis}

The analysis focuses on $V(Z / W) H \rightarrow V(W / Z) W W$ [3] processes requiring three or four isolated leptons with $p_{\mathrm{T}}>10 \mathrm{GeV}$ and a charge sum of \pm 1 . For the three leptons final state, two signal regions (SR) are designed to enhance the sensitivity: one with at least one same flavour opposite-sign (SFOS) lepton pair and one without. The signal region with SFOS lepton pairs is called $Z$-enriched sample, since the $Z$ process represents an irreducible background. The one without SFOS lepton pairs is called Z-depleted sample. To suppress $t \bar{t}$ background, $N_{\text {jets }} \leq 1$ and $N_{b-j e t}=0$ cuts are applied. A selection is also applied on the $E_{\mathrm{T}}^{\text {miss }}$ and $m_{l l}$ in order to reject $Z+$ jets background. To enhance the signal, a cut is applied on the angular correlation between leptons from 


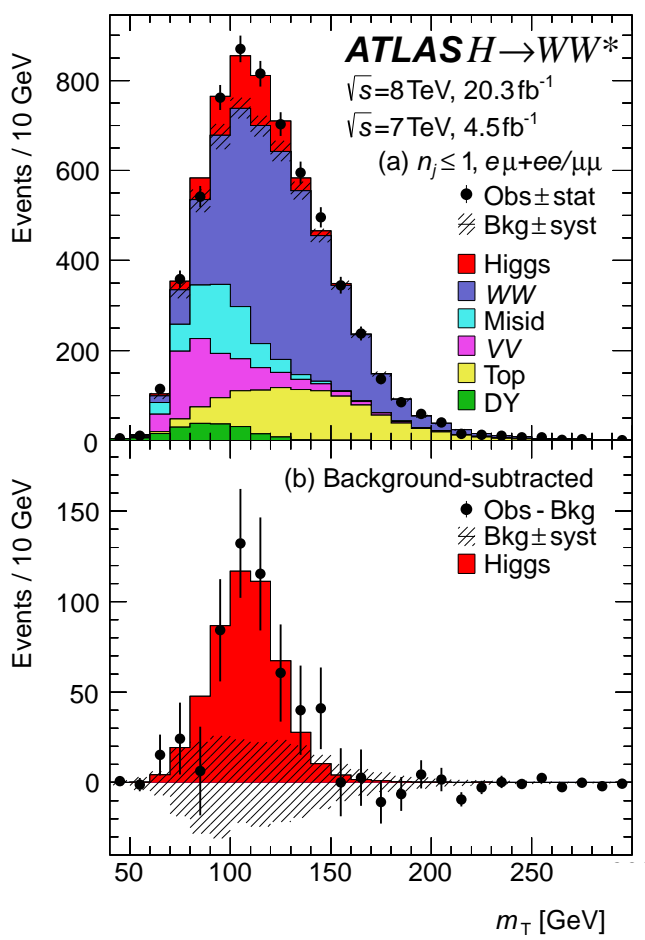

(a) $\operatorname{ggF}$

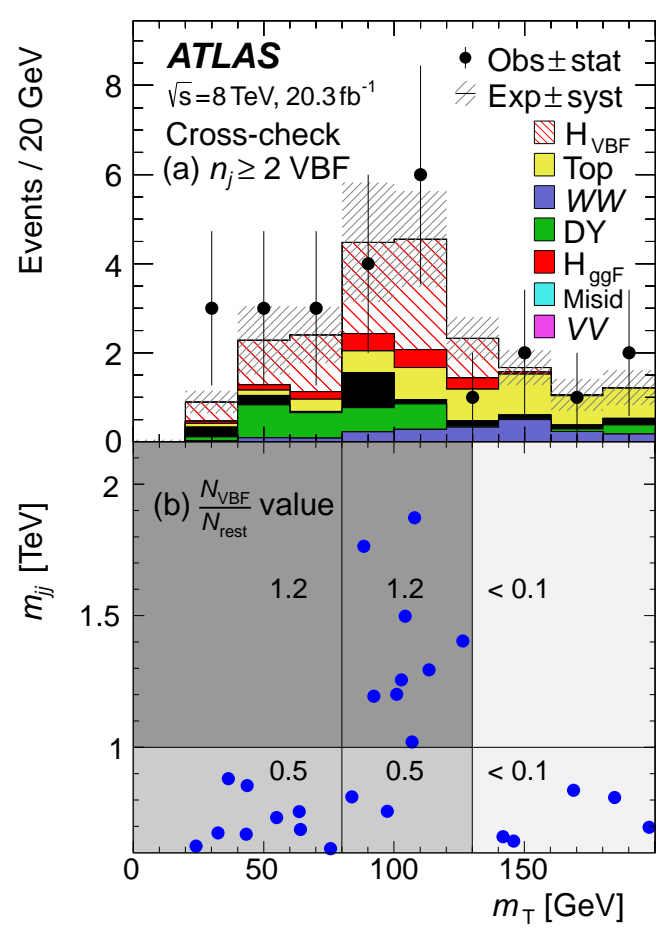

(b) VBF

Figure 2: 2a Postfit combined transverse mass distributions for $N_{\text {jet }} \leq 1$. The bottom part of the plot shows the residuals of the data with respect to the estimated background compared to the expected distribution for an SM Higgs boson. 2b Postfit distributions in the cross-check analysis in the VBF-enriched category: the upper part of the plot shows $m_{\mathrm{T}}$ and the bottom $m_{j j}$ versus $m_{\mathrm{T}}$ scatter plot for data. [2]

the Higgs candidate, $\Delta R_{l l}$. The main backgrounds in the analysis are WZ, Z+jets and top-quark. The background prediction relies on the Monte Carlo simulation with normalisation factors derived in dedicated CRs. The WZ CR is defined with inverted $Z$-mass veto and $\Delta R_{l l}$ cut in the $Z$-enriched sample. By inverting the $Z$-mass veto and the $E_{\mathrm{T}}^{\text {miss }}$ cut, it is possible to define a $Z+j$ ets $C R$ for the $Z$-enriched category. The top-quark CRs are defined by requiring at least one $b$-tagged jet in both $Z$-enriched and $Z$-depleted SR. Fig. 3a shows the $\Delta R_{l l}$ distribution in the $Z$-depleted SR, which is used as discriminant variable in the statistical analysis.

For the four leptons final state, the $p_{\mathrm{T}}$ of the leading and sub-leading leptons must be above $25 \mathrm{GeV}$ and $20 \mathrm{GeV}$, respectively, and the $p_{\mathrm{T}}$ of each of the remaining two leptons must exceed $15 \mathrm{GeV}$. The total charge of the four leptons is required to be zero. Only events with at least one SFOS lepton pair are accepted, and events are assigned to the 4l-2SFOS and $4 l$-1SFOS SRs according to the number of such pairs. The sensitivity is improved by exploiting two additional variables: the azimuthal $\left(\Delta \phi_{l_{0} l_{1}}^{\text {boost }}\right)$ angle between the two leptons from the Higgs boson candidate in the frame where the Higgs boson's $p_{\mathrm{T}}$ and the vector sum of the lepton transverse momenta. The main backgrounds contributing to the $4 l$ SRs are diboson processes, dominated by $Z Z^{*}$ and triboson processes, in particular $Z W W^{*}$, which mimics the signal. To normalise $Z Z^{*}$, a dedicated 
CR is defined. Fig. 3 b shows the distribution of $\Delta \phi_{l_{0} l_{1}}^{\text {bost }}$ in the $4 l$-1SFOS SR.

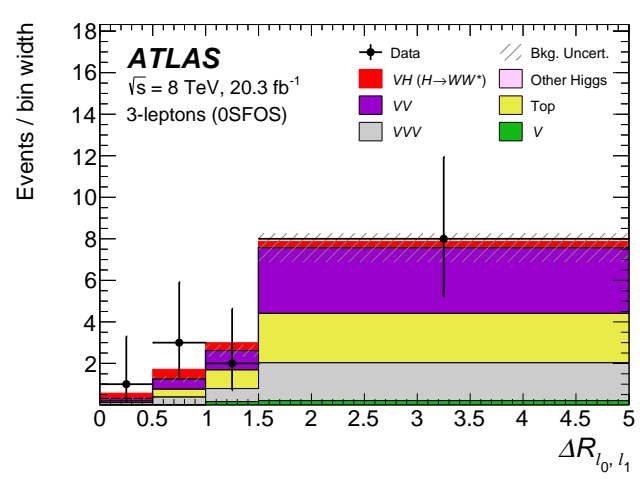

(a) $3 l$-OSFOS

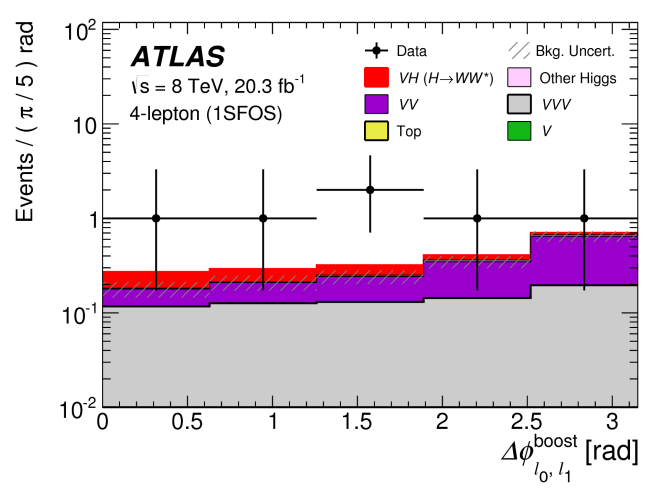

(b) $4 l$-1SFOS

Figure 3: 3a The angular separation of the two opposite-sign leptons in the 3l-0SFOS SR. 3b Distributions of $\Delta \phi_{l_{0} l_{1}}^{\text {boost }}$ in the $4 l$-1SFOS events. [3]

The leading systematic uncertainties in this analysis are coming from lepton efficiency, jet energy scale and resolution, and extrapolation of the top-quark normalisation factor from the CRs to the signal regions for the $W H$ channel. For a Higgs boson mass of $125.36 \mathrm{GeV}$, the observed (expected) deviation from the background-only hypothesis, corresponds to a significance of 2.5 standard deviations. The signal strength of the $V H$ production mode is found to be $\mu_{V H}=3.0^{+1.3}-1.1$ (stat. $)_{-0.7}^{+1.0}($ sys. $)$. The combination with the gluon fusion and VBF analyses gives an observed significance for a Higgs boson decaying in $W W^{*}$ of 6.5 standard deviation for a SM Higgs boson.

\section{Differential cross section}

The measurements of fiducial and differential cross sections for Higgs boson production in the $H \rightarrow W W^{*} \rightarrow l v l v$ final state has been performed using the $20.3 \mathrm{fb}^{-1}$ of data collected at $8 \mathrm{TeV}$ [4]. The results characterize the gluon fusion production mode, which is the dominant production mode.

The differential ggF Higgs boson production cross sections are chosen to probe several physical effects, such as the higher-order perturbative QCD contributions, multiple soft-gluon emission and the parton distribution functions.

This analysis is an extension of the ggF coupling measurement presented in Sec. 2. However, the analysis is looking only at events with two leptons of different flavour and use a simplified approach to extract the signal yields. Differential fiducial cross sections are measured in bins of the jet multiplicity $\left(N_{j e t}\right)$, the Higgs boson transverse momentum $\left(p_{\mathrm{T}}^{H}\right)$, the rapidity of the dilepton system $\left(\left|y_{l l}\right|\right)$ and the transverse momentum of the leading jet $\left(p_{\mathrm{T}}^{j_{1}}\right)$ distributions.

The measured differential cross section as a function of $N_{\text {jet }}$ is presented in Fig. $4 \mathrm{a}$ and as a function of $p_{\mathrm{T}}^{H}$ in Fig. $4 \mathrm{~b}$. The results are compared to particle-level predictions from Powheg NNLOPS [5], Sherpa [6], and MG5 aMC@ NLO [7] for the ggF production mode. In addition, the results for the $N_{\text {jet }}$ distribution are compared to the parton-level BLPTW [8] calculation, combining the NNLO+NNLL-accurate inclusive and the NLO+NLL-accurate inclusive $H+1$-jet cross 
sections, including resummation in the covariance matrix. The measured distributions agree with the predictions within the uncertainties.

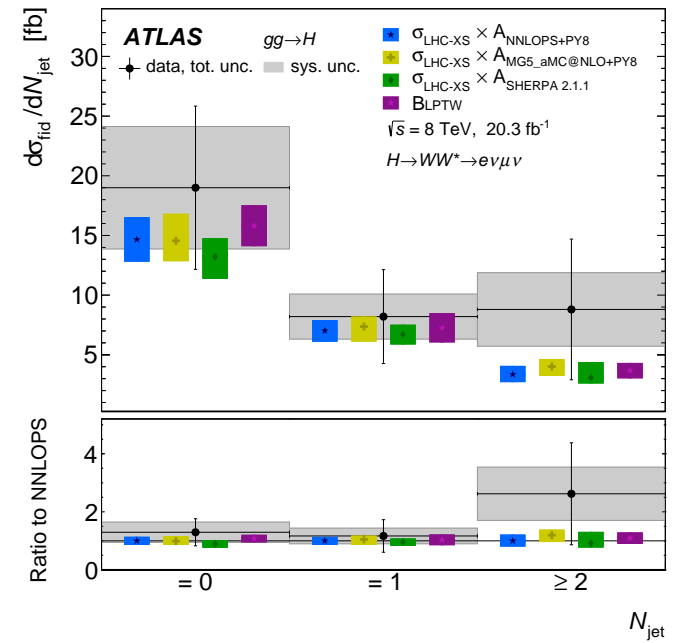

(a) $N_{\text {jet }}$

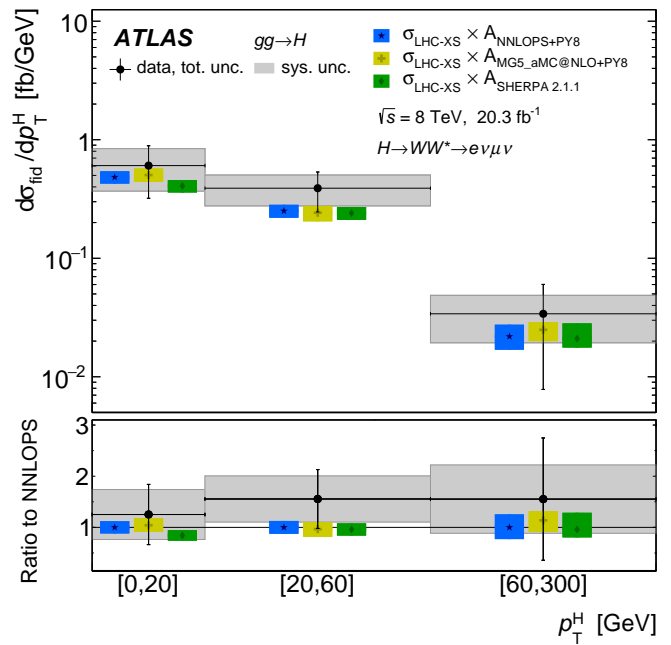

(b) $p_{\mathrm{T}}^{H}$

Figure 4: Measured fiducial differential cross section as a function of $4 \mathrm{a} N_{j e t}$ and $4 \mathrm{~b} p_{\mathrm{T}}^{H}$ overlaid with the signal predictions [4]. The ratios of the results to the predictions are given in the lower panel of each figure. The measured results are compared to various theoretical predictions.

The dominant systematic uncertainties are in the background model, in particular the MC modelling of top-quark and $W W$ backgrounds. Moreover, the uncertainties from the experimental inputs are also non-negligible

The fiducial cross section of ggF Higgs boson production is measured to be $\sigma_{g g F}^{f i d}=36.0 \pm$ 7.2 (stat. $) \pm 6.4$ (sys. $) \pm 1.0($ lumi $)$ fb for a Higgs boson mass of $125.0 \mathrm{GeV}$.

\section{References}

[1] ATLAS Collaboration, The ATLAS Experiment at the CERN Large Hadron Collider, JINST 3136 (2008) S08003.

[2] ATLAS Collaboration, Observation and measurement of Higgs boson decays to $W W^{*}$ with the ATLAS detector, Phys. Rev. D, 92, 1, 2015, doi="10.1103/PhysRevD.92.012006".

[3] ATLAS Collaboration, Study of $(W / Z) H$ production and Higgs boson couplings using $H \rightarrow W W^{*}$ decays with the ATLAS detector, Journal of High Energy Physics, 2015,1029-8479, doi="10.1007/JHEP08(2015)137".

[4] ATLAS Collaboration, Measurement of fiducial differential cross sections of gluon-fusion production of Higgs bosons decaying to $W W^{*} \rightarrow e v \mu v$ with the ATLAS detector at $\sqrt{s}=8 \mathrm{TeV}$, Journal of High Energy Physics, 2016, doi="10.1007/JHEP08(2016)104".

[5] K. Hamilton, P. Nason, E. Re and G. Zanderighi, NNLOPS simulation of Higgs boson production, JHEP 10 (2013) 222, arXiv:1309.0017

[6] T. Gleisberg et al., Event generation with SHERPA 1.1, JHEP 02 (2009) 007, arXiv:0811.4622 
[7] J. Alwall et al., The automated computation of tree-level and next-to-leading order differential cross sections and their matching to parton shower simulations, JHEP 07 (2014) 079, arXiv:1405.0301

[8] R. Boughezal, X. Liu, F. Petriello, F.J. Tackmann and J.R. Walsh, Combining resummed Higgs predictions across jet bins, Phys. Rev. D 89 (2014) 074044, arXiv:1312.4535 